\title{
Introduction to the thematic issue on stress, pain and the brain
}

\section{Conceptualizing rehabilitation as facilitated allostasis and the mitigation of uncertainty}

\author{
Gary Goldberg $^{\mathrm{a}, *}$, Blessen Eapen ${ }^{\mathrm{b}, \mathrm{c}}$ and Leonard Kamen ${ }^{\mathrm{d}, \mathrm{e}, \mathrm{f}}$ \\ ${ }^{\mathrm{a}}$ Department of Physical Medicine and Rehabilitation, Medical College of Virginia, Virginia \\ Commonwealth University Healthcare System, Richmond, VA, USA \\ ${ }^{\mathrm{b}}$ Department of Medicine, Division of Physical Medicine and Rehabilitation, David Geffen \\ School of Medicine at UCLA, Los Angeles, CA, USA \\ ${ }^{\mathrm{c}}$ Physical Medicine and Rehabilitation Service, VA Greater Los Angeles Health Care System, \\ Los Angeles, CA, USA \\ ${ }^{\mathrm{d}}$ MossRehab Hospital, Albert Einstein Healthcare Network, Philadelphia, PA, USA \\ ${ }^{\mathrm{e}}$ Adjunct Associate Professor, Department of Physical Medicine and Rehabilitation, \\ Temple University Hospital, Philadelphia, PA, USA \\ ${ }^{\mathrm{f}}$ Department of Physical Medicine \& Rehabilitation, Thomas Jefferson University Hospital, \\ Philadelphia, PA, USA
}

\section{Introduction}

We provide a general introduction to this thematic issue of NeuroRehabilitation on the theme of 'stress, pain and the brain' including general background as well as an exploration of the tenets of existential stress medicine and how they draw brain injury and pain into a common rubric centered on the concept of the brain as the primary body organ involved in strategizing to address uncertainty. It is argued that this conceptual framework provides the opportunity to integrate 'bottom-up' analytical studies from conventional neuroscience with 'top-down' organismic examination of the whole person as an embodied self-actualizing agent (Goldstein 1939) embedded in a complex environmental context, whose primary activity involves the anticipatory, predictive pro-

\footnotetext{
*Address for correspondence: Gary Goldberg, Physical Medicine and Rehab. Service (117A), McGuire VA Medical Center, 1201 Broad Rock Blvd., Room 2A-140, Richmond, VA 23249, USA. Tel.: +1 804675 5000; E-mail: gary.goldberg.md@gmail. com.
}

cess of allostatic orchestration (Lee 2019) directed toward minimizing free energy, or uncertainty (Friston 2010). In this general context, we propose that a common underlying pathophysiological process is associated with the functional impact of brain injury and can explain the tendency, under conditions of chronic allostatic overload, toward the co-existence of various stress-related disorders, including, but certainly not limited to, chronic pain. Following this general introduction to the underlying theme of this thematic issue, and how we regard its significance, we provide specific introductions to each of the six papers that were contributed and are published together here. We then summarize and derive inferences regarding the implications of this work for brain injury rehabilitation, and provide some general thoughts regarding future directions.

\section{Background}

As we write an introduction to this thematic issue of NeuroRehabilitation, we are now well into the third 
month of social distancing isolation in the United States in an attempt to slow down the rise in devastation and loss of life from the global pandemic of COVID-19 due to the SARS-CoV-2 Corona virus. We are in the throes of a global viral pandemic that has now taken the lives of over 100,000 in the USA and over 400,000 worldwide. We are also well into a global economic downturn that has created levels of unemployment not seen since the Great Depression and significant economic uncertainty. In addition, most recently, in multiple cities across the United States and in countries around the world, there have been massive protest marches and crowds gathered to demonstrate against systemic state-supported racism and social injustice triggered by the intentional killing of an unarmed African-America man while in the custody of a group of Caucasian police officers in Minneapolis, Minnesota. The disturbing sounds of the sirens of multiple ambulances, fire trucks and police vehicles rushing to address emergent situations of various kinds have become increasingly commonplace in urban centers around the world. In other words, we are now experiencing massive global dislocation and significant uncertainty that is clearly precipitating ubiquitous and widespread stress among large numbers of human beings around the world.

When we began the process of planning for this issue over two years ago, the possibility that such a situation could potentially arise was remote and certainly not what the majority of the population was prepared for, though the emergence of the situation was conceivable given a prior accelerating history of global viral pandemics. However, the current situation is not something for which one could have readily prepared nor something for which the detailed impact-psychologically and in terms of general physical health-could have been readily anticipated. In effect, though, it is a clear demonstration on a massive scale of the general principle that prompted the planning of this issue. Namely, the existential connection between stress precipitated by uncertainty and the risk of this situation becoming chronic if uncertainty cannot be definitively resolved, chronic pain syndromes, mood disorders, sleep disturbance and brain injury. This approach derives from recognizing the primary function of the human central nervous system for directing energy flows and strategizing the reaction, at all levels of physiological response, to the challenge of uncertainty regarding the future and what it may bring in the way of existential confrontation (Joëls \& Baram 2009)—in the process of grappling with mortality and finitude, and dealing with the challenges to maintaining momentto-moment viability of life-sustaining processes that emanate both from within and from outside the organism (Peters, McEwen \& Friston, 2017).

Two of us (GG and BE) were involved as co-authors on a paper that examined the interrelationship between brain injury and chronic pain, along with several other associated conditions (Hoot, Levin, Smith et al. 2018). This study examined the effects of mild traumatic brain injury (mTBI) in a US military population on pain intensity and pain interference and showed that mTBI was strongly associated with both of these measures of pain when comparing veterans with and without a history of mTBI. However, there was also convincing evidence of mediation of the interaction between mTBI and chronic pain through multiple common chronic mTBI co-morbidities, sometimes linked together in the condition known as 'postconcussion syndrome', including posttraumatic stress disorder (PTSD), depression, anxiety and sleep disturbance. In thinking about the demonstrated clustering of these chronic clinical symptoms and conditions, we began to wonder about the possibility of a common underlying pathophysiological mechanism tying it all together. There is, in particular, a clear overlap between symptomatology linked to postconcussion syndrome after mTBI and to chronic pain phenotypes (Nampiaparampil 2008; Irvine \& Clark 2018; Mollayeva et al. 2017; Grandhi et al. 2017; Mills, Nicholson \& Smith 2019) both of which may be associated with an increased incidence of comorbid mood disorders, generalized fatigue, motor dysfunction, sleep disturbance and specific cognitive deficits related to frontal lobe injury, typically producing faulty executive function, as well as subtle impairments in corticolimbic processing and emotional regulation (Vachon-Presseau et al. 2016). How are we to understand this broad overlapping of symptomatology? Studies of individuals with chronic pain have shown elevated AM cortisol levels, smaller hippocampal volume and enhanced phasic responses to affectively provocative stimuli in the parahippocampal gyrus on functional magnetic resonance imaging (fMRI) as compared to control subjects without chronic pain, suggesting that a stress model of pain linked to specific corticolimbic, hypothalamic-pituitary-adrenal (HPA) axis and neuroendocrine dysfunction is associated with increased vulnerability to persistent chronic pain states (Vachon-Presseau, Roy, Martel et al. 2013). 
What might well explain these clinical correlations would be a common chronic state of hyper-arousal or enhanced reactivity linked to the development of a state of chronic stress - a state of chronic 'allostatic overload'-associated with a broad range of interconnected phenomena in the brain and body (Joëls $\&$ Baram 2009). One particular anatomic region of the brain in which chronic stress can have significant influence and the stress-related impairment of which may well be linked to a disordering of both executive function and corticolimbic regulation of affect, is the prefrontal cortex (Girotti, et al. 2018).

We became interested in looking further into the possibility of utilizing a stress model in association with an understanding of the behavioural implications of mTBI and the clustering of stress-related conditions that overlap between chronic pain and mTBI. Might there be a common thread linking these conditions together? One may hypothesize a common pathophysiological substrate in prefrontal cerebral cortex related to executive function impairment, impaired regulation of affect linked to corticolimbic alteration, and associated neuroendocrine dysfunction in the response to chronic stress or, equivalently, to the persisting 'allostatic load'-where 'allostasis' is defined as the circular continuous process of adaptive response to changing conditions so as to adjust and maintain life-sustaining physiological processes within operable range in the face of such challenge, thus attaining 'stability through change' (Ganzel, Morris \& Wethington, 2010; Sterling \& Eyer, 1988; Sterling, 2012, 2014; Lee 2019)—that might then predispose individuals with mTBI to the development of chronic pain along with the co-morbid mediating chronic symptomatology and conditions that had been observed.

\section{General theoretical foundation}

Based on his work with nearly two thousand braininjured German soldiers after World War I, Kurt Goldstein (1939) introduced a holistic organismic approach to understanding the functional effects of brain injury on the whole person as singular selfactualizing agent, and how to approach its treatment. Among other things, he brought attention to the concept that what is observed in the behavior of a person after TBI is a complex overlay of the direct effects of injury together with the attempts of the injured person to adaptively cope and compensate for the effects of injury to the extent they are able to do so, so as to continue to be capable of self-actualization, although in a state of reduced functional range due to the residual effects of the injury, especially on abstract thinking ability. Goldstein also noted that when the adaptive compensation was not adequate to the demands of a challenging cognitive task, what he called a 'catastrophic reaction' may be precipitated. This occurrence of a disabling anxiety-fed disordering of behavior associated with irresolvable subjective uncertainty regarding the capacity to adequately meet existential requirements given the consequences of brain injury, suggests the unmasking of the chronic disabling stress associated with this existential uncertainty that can rapidly break through the defensive barriers constructed to manage it. Given that the brain is the primary organ involved in orchestrating the management of uncertainty (Friston 2010; Peters, McEwen, \& Friston 2017; Lee 2019), the effect of the injury is essentially two-fold: the injury itself precipitates significant distress linked to uncertainty regarding the pragmatic impact of the injury in daily life, and it also, at the same time, impairs the ability to strategize effectively so as to address the uncertainty that the injury itself has precipitated.

Friston (2010) proposed the concept of the 'free energy principle' which sees the main overall goal of the central nervous system to be the minimization of 'free energy' in the organism-environment system as detected in the sensory states of the organism, thus reducing the risk of disorder and fragmentation due to uncertainty regarding future conditions with perceived existential significance. Through processes that permit accurate anticipation of future contingencies, the organism utilizes cerebral energy flows to support anticipatory strategizing to address and reduce uncertainty and to limit surprise. The brain interacts with all facets of the body to coordinate and selectively deploy energy to coordinate the anticipatory engagement (Lee 2019). This energy-consuming process of addressing the need to mitigate uncertainty through adaptive strategizing is called 'allostasis' - the achievement of viable stability through adaptive change, which is distinguished from 'homeostasis' wherein reactive feedback loops operate to keep key state parameters stable. While allostasis involves active predictive strategizing by the central nervous system to adaptively manage complex environmental and pathology-linked challenges, homeostasis tends to refer to keeping the internal environment of the body close to stable setpoints (Lee 2019). The conditions which introduce uncertainty and thus precipitate an energetic demand 
for the adaptive allostasis, constitute the 'allostatic load', also understood as 'stress.' Ideally, allostasis is effective in anticipating and addressing future contingencies, and the acute stress raised by the uncertainty abates or is adequately mitigated with a successful predictive approach - the allostatic load is efficaciously addressed. However, there are often situations in which the response is incomplete and allostatic load persists and must be continuously addressed and managed over time. For many different possible reasons, the inherent uncertainty of the situation cannot be fully addressed and defused, and one is left in an ongoing situation of chronic allostatic load to which attention (and energy) must be continuously directed (McEwen 2017). Alternatively, the particular coping strategy to which an individual turns, often due to maladaptive tendencies previously formed during prior challenging life experience(s), may actually be either ineffective in addressing the allostatic load, or may potentially exacerbate and perpetuate it. The effects of the injury itself may significantly interfere with the ability to mount effective coping strategies. Over time, there may be a gradual habituation of response-which tends to occur more readily in some individuals ('habituators') compared to others ('non-habituators'), but, there may also be a situation of continuing 'non-habituation' in which the stress continues and becomes corrosive over time, consuming energy and wearing down tissues, creating vulnerability to the accumulation of other stress-related conditions that either further contribute to the precipitation of, or are precipitated by, the unabated chronic stress (Peters, McEwen, Friston 2017). With these concepts, one can begin to formulate a stress model of the impact of mTBI that can provide an explanation for the clustering of the observed conditions mediating the interaction between mTBI and stress-associated chronic pain (Vachon-Presseau 2018; Lunde \& Sieberg 2020) as well as other stress-related conditions seen in higher frequency after mTBI, including mood disorders (McEwen 2003; Barrett, Quigley \& Hamilton 2016; Strain 2018; Hill, Hellemans, Verma, et al. 2012) and sleep disturbance (McEwen 2006; Juster \& McEwen 2015; McEwen \& Karatsoreos 2015; Wickwire et al. 2018).

In a seminal paper, Peters, McEwen \& Friston (2017) examined the linkage between the issue of stress and uncertainty and proposed an 'informationtheoretic approach' based upon the 'free energy principle' (Friston 2010) which holds that selforganizing biological agents (like human subjects) actively resist a prepotent tendency toward disorder and fragmentation, and, to do so, they must work to minimize the uncertainty or 'free energy'/entropy in the organism-environment system as detected through sensory channels through cognitive strategizing and anticipatory engagement directed through the central nervous system, a process which can consume significant amounts of available energy in support of cerebral function. If, in spite of all best efforts, significant uncertainty persists, either due to challenging environmental contingencies or brain-body functional limitation, for example, due to effects of injury, then a state of chronic stress ensues which can lead to persisting cerebral insufficiency and a state of chronic allostatic overload that can then lead to various chronic systemic pathologies as well as stress-linked symptoms such as chronic pain, mood disorder, fatigue, posttraumatic stress and sleep disturbance which may also be associated with impairment of cerebral functionality and contribute to worsening of the overall energetic crisis. Peters, McEwen \& Friston (2017) then go into a detailed examination of various brain systems involved in the process of avoiding surprise and addressing the uncertainty associated with allostatic load.

Lee (2019) has proposed a further elaboration of the concept of allostasis in what he has termed the 'Paradigm of Allostatic Orchestration' (PAO) seen as a detailed extension of the concept of allostasis that places emphasis on the role of the brain and brainbody-system 'facets' in the coordinated anticipatory response conducted by the central nervous system in concert with body-based systems in response to allostatic load. In addressing questions of treatment of states of chronic allostatic overload and the associated energetic crisis that can be precipitated, Lee notes three levels of potential therapeutic intervention: (1) environmental, social and/or intersubjective relational buffering of stress, recognizing that social environment can have both positive and negative influences on allostatic load management (see also: McEwen 2012), (2) intrapersonal in terms of training in and incorporation of various effective stress management practices, and (3) direct physiological and/or neuro-psychopharmacological treatments that serve to enhance the brain's ability to manage allostatic load. One example of an innovative approach in this realm is the application of neuromodulation technology (Gerdes et al. 2013; Tegeler et al. 2016; Shaltout et al. 2018).

Not everyone experiences the inevitable development of allostatic overload and the diminishing and 
disabling consequences and chronic pathology associated with chronic stress. Psychobiological allostasis can confer both stress resilience as well as vulnerability (Karatsoreos \& McEwen 2011). Stress associated with adverse experience does have a clearly beneficial impact in some individuals, becoming an opportunity for the experience of posttraumatic growth (Tedeschi \& Calhoun 1995). The experience of stress may also engender beneficial learning associated with induction of brain plasticity (McEwen \& Gianaros 2011). In a systematic review and meta-analysis of post-traumatic growth after acquired brain injury, Grace et al. (2015) state that 'various demographics, injury-related variables, subjective beliefs and psychological health are related to (posttraumatic) growth.' In conceptualizing mTBI and these related stress-associated conditions as the result of chronic allostatic overload, the question arises regarding how one might best prevent this state from developing or, even more valuable, if effective therapeutic strategies can be found to transform the experience of stress, seeking to sort out how to alter the impact and course of chronic stress away from diminishment of health and the seemingly inevitable induction of chronic metabolic pathology, and toward the transformative potential of posttraumatic growth and the development of stress-resistant resilience. How stress is managed and possible brain mechanisms that may lead to the development of resilience rather than pathology is a subject of significant research interest (eg. see: Worley, Hill \& Christianson 2018). There is accumulating evidence that subjective beliefs regarding the meaning to be drawn from experience and the cognitive set or 'appraisal' that an individual has with respect to the relationship with stressful activity and how the stress is thus perceived can have significant influence on how stress is experienced and processed (Crum et al. 2017). The stress may be perceived as threatening when a person evaluates the environmental demands as outweighing their own available resources and ability to cope. An awareness of relative vulnerability to deleterious effects of stressful experience related to self-perceived cognitive limitation after brain injury may be one reason why stress reactivity may be enhanced following mTBI, although enhanced stress reactivity may also be one of the direct physiological consequences of the injury (Griesbach et al. 2011; Hoffman \& Taylor 2019). Furthermore, the observation that attentional responses evoked by emotionally provocative stimuli are enhanced after mTBI (Mäki-Marttunen et al. 2015), may reflect increased reactivity and altered processing of affect-evoking stimuli in corticolimbic pathways, which has also been shown to be correlated with susceptibility to the development of chronic pain syndromes (Vachon-Presseau et al. 2013; VachonPresseau et al. 2016; Vachon-Presseau 2018).

\section{Introduction to contributed papers}

The editors solicited research papers related to the general theme of the issue and six papers covering a broad range of topics generally related to the theme were submitted, reviewed, and have been included in this collection. The guest editors would like to take this opportunity to thank each of the authors involved for agreeing to submit their work for inclusion in this thematic issue of NeuroRehabilitation.

Walsh et al. (2020) provide a detailed case report through recursive application of interpretive phenomenological analysis (IPA) to examine the impact of stress and stress-related processing as common sequelae of TBI. A key theme emerging from the analysis identifies the crucial impact of 'uncertainty' in the process of reconstituting the relational life-world of the survivor of a significant TBI. This approach actually becomes a pragmatic and valuable exercise detailing an insightful introspective narrative that lends itself to IPA and the drawing out of key themes based on analysis of a structured interview. Social context is shown to play a key role in the process of adjustment, coping and well-being in an articulate, thoughtful individual confronted with the task of reconstituting a sense of self and a narrative through which he is able to make sense of and draw constructive meaning from the repercussions of a significant TBI in the setting of a relational social network that includes family, friends and work associates. Emerging themes, including dealing with paradox, adjusting perspectives and responding to the stress precipitated by the uncertainty that emerges after a significant TBI, are examined. The struggle to regain a coherent sense of self and social identity is evident and magnified by chronic pain interference. The authors convincingly make the point through their examination of this case, that the enterprise of rehabilitation after TBI cannot be adequately undertaken without fully recognizing the importance of an inter-and intrasubjective relational perspective that fully honors the subjective being not only of the identified injured person seeking assistance, but also of those others closely connected through various forms of communicative interaction in their social network who are also sig- 
nificantly impacted. The issue of the uniqueness of TBI in this context, recognizing the implications of the fact that the injured brain-particularly, as in this case, when significant frontal lobe damage is incurred-also has a central role to play in the anticipatory allostatic response to the precipitated uncertainty, is also nicely demonstrated.

The manner in which an individual copes with stress may turn out to be adaptive with a beneficial impact leading to resolution, or it may turn out to be maladaptive with the resultant precipitation of a debilitating state of chronic allostatic overload that can place the injured person at risk for mood disorder and other chronic stress-related conditions. The whole question of the response to TBI in terms of how a particular individual may cope with resultant stress and whether their choice proves effective or not is a fascinating and important issue that may lead to the early identification of individuals at risk for development of depression and anxiety as complications of unabated chronic allostatic overload precipitated by the injury. Certain personality traits may also increase vulnerability to the development of depression. This raises the possibility of developing and applying early intervention strategies for individuals thus identified to be at increased risk for developing depressive symptoms following TBI. This is the general question that is addressed by McIntyre et al. (2020a) in the first of their two papers included here. They report an observational cohort study examining coping strategies and personality traits among individuals with acquired brain injury $(\mathrm{ABI})$ and the extent to which these correlate with reports of significant symptoms of depression. Those reporting symptoms of depression were significantly more likely to manifest evidence of experiential avoidance implying that attempting to limit allostatic load through avoiding potentially stressful experience paradoxically ends up placing one at increased risk for depression. McIntyre et al. also report a significant interaction between reported depressive symptomatology and the combination of coping strategy type and personality traits and then drill down on which specific coping strategies and personality traits were most likely to correlate with increased depressive symptomatology. Another interesting finding with potential implications for therapy is that those reporting depressive symptomatology rarely turned to problem-based active coping strategies suggesting that these strategies may actually provide, or possibly reflect, an adaptive beneficial effect.
McGeary et al. (2020) report a study performed as a secondary analysis of data collected from 93 veterans who presented with the combination of chronic pain, head injury, posttraumatic stress and long-term opioid use in order to seek evidence for the 'Fear Avoidance Model' (Edwards et al. 2016) of chronic pain through examination of risk factors linked to fear avoidance that include pain catastrophizing, fear avoidance beliefs, anxiety and depression. The Fear Avoidance Model proposes that pain-related disability is precipitated by an interacting, cyclical sequence of fear-related cognitive, affective and behavioral processes with the basic idea being that it is the fear of pain and the reluctance to do anything that could elicit the pain which then leads to a self-reinforcing cascade of negative consequences. The examination of the data performed by McGeary et al. provides strong evidence for the Fear Avoidance Model as an explanation for the development of chronic pain in this population with a total of nearly $40 \%$ of the variance in pain-related disability accounted for by measures of the four components constituting the model with each of the four factors contributing a $6 \%$ increase in disability. Of significant interest, particularly in view of the findings reported here by McIntyre et al. (2020a; 2020b) with respect to the significant deleterious impact of experiential avoidance, is the potential role of fear avoidance as a maladaptive response to concomitant TBI as a contributing factor to the development of chronic pain states in individuals with TBI, given that fear avoidance has also been found to be a potential explanation for persistent symptoms following TBI (Wijenberg et al. 2017).

McIntyre et al. (2020b), in their second paper contributed to this issue, focus on the subgroup of individuals with the combination of high levels of anxiety sensitivity (AS) and experiential avoidance (EA) as important factors associated with reports of depressive symptomatology, increased anxiety and poorer quality of life after ABI. They found that their experimental population could be subclassified into three unique subgroups such that AS and EA were significantly different between the groups: a group with generally low AS and EA, a second group with moderate levels of AS and EA and a third group with high levels of AS and EA. The group with the lowest AS and EA reported a quality of life that was significantly higher than the third group with the highest AS and EA. They suggest that the early identification of individuals in this third group of high AS and EA as being at high risk for high levels of anxiety, depressive 
symptomatology and poorer quality of life may be helpful in initiating intensive interventions directed to the improvement of mood and associated quality of life. These individuals may well be the group of 'non-habituators' identified by Peters, McEwen \& Friston (2017) as those who are particularly prone to the development of chronic allostatic overload and the accumulation of chronic stress-related conditions that accompany this situation. A variety of different interventions have been proposed to manage and assist this population and McIntyre et al. (2020b) review evidence supporting the efficacy of a number of these in the discussion section of their paper. Having in place effective methods for early identification of such individuals so that they can be supported and treated with effective interventions for the facilitation of allostasis, instruction in self-management strategies for reducing and managing anxiety, and, to the extent possible, assistance with the mitigation of the uncertainty driving the anxiety, would appear to be a justifiable goal and the subject of future research. The possibility of addressing and countering the beliefs that underly experiential avoidance through social engagement, individual psychotherapy, mindfulness and relaxation techniques, and psychobiological methods directed toward the relaxation and 'letting go' of the fear that reinforces avoidance-related beliefs requires further exploration and development in this identified population. The facilitation of active coping oriented around effective pragmatic problem-solving may be of significant benefit. The discrete and judicious deployment of psychedelic-supported psychotherapy for the facilitation of active coping and the 'letting go' of the underlying beliefs supporting intractable anxiety may be another avenue open for careful and prudent research exploration (Carhart-Harris \& Friston 2019; Wolff et al. 2020).

One of the potentially most devastating effects of TBI is the manner in which it may cause significant impairment of communication and social skills that are necessary for successful community re-integration, return to gainful employment, and the capacity to become a valued member of a social group and a contributing member of society, among other important practical everyday activities. Impairment in social functioning can isolate an individual from the potential support of others and the validation that positive social relationships can offer. The negative fall-out from impairment of social communication skills including both verbal and nonverbal components, can feed into the fear-related beliefs that sustain experiential avoidance and social withdrawal. The ability to participate in a respectful conversation, the ability to understand turn-taking in conversation (i.e. conversational pragmatics), the ability to read nonverbal cues and the ability to be able to take another's position and experience empathy for the other are all critically important social skills that can be significantly degraded due to the effects of TBI. Raukola-Lindblom, Vuorinen \& Variainen (2020) provide an overview and an assessment of their experience with a community-based therapeutic group in Finland focused on the improvement of communication and social skills in individuals recovering from TBI. This is clearly an example of a relational intervention that focuses on the reduction of stress and the development of comfort and basic skills involved in group interaction with critical supervision and oversight provided by experienced therapists to ensure the therapeutic nature of the interactions is prioritized and to facilitate the positive benefits of group engagement for each of the participants. As they state in their paper: "Practicing social skills in a group situation is inherently selfmotivating and encourages a constructive, positive impetus toward greater social participation." They describe an innovative, relational, socially relevant intervention that can have powerful beneficial influence with their groups providing the foundation for participants to build potentially long-lasting, valuable relationships with peers and, in the process, to reconstruct a positive social identity and self-concept that may have been significantly challenged by the often subtle and complex negative effects of the injury that only become evident in social context.

The final contributed paper addresses a constant question that comes up in the management of patients with MTBI which is the tremendous variability in symptom production associated with the injury that does not appear to directly correlate with either the severity or the nature and localization of the injury. Why do some individuals with an MTBI have a minimal and short-lasting set of symptoms while others may experience intractable, long-lasting and significantly disabling consequences? In a group of 80 individuals in outpatient rehabilitation treatment for symptoms associated with MTBI, Esterov et al. (2020) generate a multivariate analysis linking a variety of potential predictor variables to the Neurobehavioral Symptom Inventory that documents the ongoing symptoms the patients are experiencing and reporting. The findings support the idea that it is not the direct effects of the injury that are neces- 
sarily the dominant factor in the development of associated disability, but rather how the person is coping with the socially dislocating effects of the injury-for example, the impact on the ability to participate in community functions. While there are several limitations recognized, including the fact that the study sample is drawn from a group of relatively non-diverse treatment-seeking individuals which, by definition, leaves out the population of those individuals whose symptoms subsided without specific treatment, the takeaway message is that the impact and disability related to the injury has less to do with the details of the injury pathology than it has to do with the difficulties the occurrence of the injury generates in the psychosocial holistic context of the injured person's life and their difficulty with coping with the resultant fall-out. Significant numbers of individuals in the sample also reported associated co-morbidities that could be understood as having a significant contributing chronic stress-related element, such as mood disorder, chronic pain and sleep disorder.

\section{Conclusion}

The integration of a 'top-down' holistic perspective that views a person as a self-actualizing, self-perpetuating organismic agent operating in a relational context to respond to allostatic load adds the potential to derive insights of significant value and clinical importance. It also provides an essential functional context for understanding the operation of neurological and various other bodily mechanisms as elucidated by 'bottom-up' analytic study using conventional bioscience. In this issue, we have assembled a group of papers that, we believe, shed important light on how this interaction may work with particular relevance to gaining insight into the relationship between stress and the experience of pain in the general context of brain impairment produced by brain injury. One insight that emerges is that how a person attempts to cope with the injury and the associated existential uncertainty it produces can have major implications for the functional consequences in reallife terms. Another related insight is that therapy must not only address the direct effects of the injury to the extent that that may be possible, but also must address dysfunctional coping precipitated by the injury. Passive coping and avoidance strategies appear to be problematic and while they may limit exposure to negative provocative experiences in the short run, in the long run, entrenched avoidance supported by the fear that emotionally provocative experience will be intolerable and self-destructive, can lead to social isolation, depression, and the burden of chronic allostatic overload. Thus, one key emerging issue is how best to assist a person with such tendencies to shift to active coping and overcome entrenched avoidance beliefs. Positive social experience in group therapy and structured individual psychotherapy, for example in the form of Acceptance and Commitment Therapy, have been advocated (see McIntyre et al. 2020b in this issue for further discussion). Psychedelic-assisted psychotherapy may be another avenue open for further research exploration in this vein.

Furthermore, one can view therapeutic approaches as operating in social-environmental relational context, intersubjective and intrapersonal context, and through direct influence on functional brain mechanisms; and that all of these contexts that influence the functionality of the injured person interact with each other in both potentially beneficial and detrimental complex ways. When examining the entire enterprise of rehabilitation for brain injury, in particular, and for all forms of neuropsychological impairment, a stress medicine orientation would promote the general idea that, to the extent possible, interventions should be designed to effectively support the beneficial aspects of allostasis, avoid the development of chronic allostatic overload and the energetic crises and accumulation of stress-related debilitating conditions, such as chronic pain, that it can precipitate, and assist the person through whatever means may be most effective, in mitigating the detrimental impact of a sense of persisting existential uncertainty. What is required is an optimal integration of insights obtained through conventional functional neuroscience-for example, with respect to neuropsychopharmacology and the operation of functional brain networks - and insights obtained through a relational, whole-person perspective that recognizes and fully honors and engages the subjective being, personality and social context of the person whose existential struggles we are obligated and entrusted to help mitigate.

\section{Acknowledgments}

The guest editors would like to dedicate this issue to the memory of Professor Bruce Sherman McEwen whose pioneering work on stress and the brain, and 
whose intellectual generosity regarding this work and his ideas, helped to lay the foundations for the concepts presented in this paper and for the theme of this issue. The guest editors would also like to thank the editors of NeuroRehabilitation and the managing editor of the journal for their support for this project throughout the process. They would also like to thank each of the authors of the six contributed papers for their interest in and support for this project.

\section{References}

Barrett, L. F., Quigley, K. S., \& Hamilton, P. (2016). An active inference theory of allostasis and interoception in depression. Philosophical Transactions of the Royal Society of London B. Biological Science, 371(1708), 20160011. https://doi.org/10.1098/rstb.2016.0011

Carhart-Harris, R. L., \& Friston, K. J. (2019). REBUS and the anarchic brain. Toward a unified model of the brain action of psychedelics. Pharmacological Reviews, 71, 316344. https://doi.org/10.1124/pr.118.017160

Crum, A. J., Akinola, M., Martin, A., \& Fath, S. (2017). The role of stress mindset in shaping cognitive, emotional and physiological responses to challenging and threatening stress. Anxiety Stress \& Coping, 4, 379-395. https://doi.org/10.3389/fpsyg.2015.01162

Edwards, R. R., Dworkin, R. H., Sullivan, M. D., Turk, D. C., \& Wasan, A. D. (2016). The role of psychosocial processes in the development and maintenance of chronic pain. The Journal of Pain, 17(9 Suppl), T70-T92. https://doi.org/10.1016/j.jpain.2016.01.001

Esterov, D., Lennon, R. J., Bergquist, T., \& Brown, A. (2020). Predictors of neurobehavioral symptom reporting in a community-based sample with mild traumatic brain injury. NeuroRehabilitation, 47, 65-77.

Friston, K. (2010). The Free Energy Principle. A unified brain theory? Nature Reviews Neuroscience, 11, 127-138. https://doi.org/10.1038/nrn2787

Ganzel, B. L., Morris, P. A., \& Wethington, E. (2010). Allostasis and the human brain: Integrating models of stress from the social and life sciences. Psychological Reviews, 117, 134-174. https://doi.org/10.1037/a0017773

Gerdes, L., Gerdes, P., Lee, L. W., \& Tegeler, C. H. (2013). HIRREM $^{\mathrm{TM}}$ : a noninvasive, allostatic methodology for relaxation and auto-calibration of neural oscillations. Brain and Behavior, 3, 193-205. https://doi.org/10.1002/brb3.116

Girotti, M., Adler, S. M., Bulin, S. E., Fucich, E. A., Paredes, D., \& Morilak, D. A. (2018). Prefrontal cortex executive processes affected by stress in health and disease. Progress in NeuroPsychopharmacology and Biological Psychiatry, 85, 161-179. https://doi.org/10.1016/j.pnpbp.2017.07.004

Goldstein, K. (1939). The Organism. A holistic approach to biology derived from pathological data in man. New York: American Book Company. Originally published in German as: Goldstein, K. (1934). Der Aufbau des Organismus: Einführung in die Biologie unter besonderer Berücksichtigung der Erfahrungen am kranken Menschen. Nijhoff: Den Haag.

Grace, J. J., Kinsella, E. L., Muldoon, O. T., \& Fortune, D. G. (2015). Post-traumatic growth following acquired brain injury: a systematic review and meta-analysis. Frontiers in Psychology, 5, 1162. https://doi.org/10.3389/fpsyg.2015.01162

Grandhi, R., Tavakoli, S., Ortega, C., \& Simmonds, M. J. (2017). A review of chronic pain and cognitive, mood, and motor dysfunction following mild traumatic brain injury: Complex, comorbid, and/or overlapping conditions? Brain Science, 7, 160. https://doi.org/10.3390/brainsci7120160

Griesbach, G. S., Hovda, D. A., Tio, D. L., \& Taylor, A. M. (2011). Heightening of the stress response during the first weeks after a mild traumatic brain injury. Neuroscience, 178, 147-158. https://doi.org/10.1016/j.neuroscience.2011.01.028

Hill, M. N., Hellemans, K. G. C., Verma, P., Gorzalka, B. B., \& Weinberg, J. (2012). Neurobiology of chronic mild stress: Parallels to major depression. Neuroscience \& Biobehavioral Reviews, 36, 2085-2117. http://doi.org/10.1016/j.neu biorev.2012.07.001

Hoffman, A. N., \& Taylor, A. N. (2019). Stress reactivity after TBI. Implications for comorbid post-traumatic stress disorder. Behavioural Pharmacology, 30, 115-121. https://doi.org/ 10.1097/FBP.0000000000000461

Hoot, M. R., Levin, H. S., Smith, A. N., Goldberg, G., Wilde, E. A., Walker, W. C., Eapen, B. C., Nolen, T., \& Pugh, N. L. (2018). Pain and chronic mild traumatic brain injury in the US military population: A Chronic Effects of Neurotrauma Consortium study. Brain Injury, 32, 1169-1177. https://doi.org/10.1080/02699052.2018.1482427

Irvine, K.-A., \& Clark, J. D. (2018). Chronic pain after traumatic brain injury. Pathophysiology and pain mechanisms. Pain Medicine, 19, 1315-1333. https://doi.org/10.1093/pm/pnx153

Joëls, M., \& Baram, T. Z. (2009). The neuro-symphony of stress. Nature Reviews Neuroscience, 10, 459-466. https://doi.org/10.1038/nrn2632

Juster, R.-P., McEwen, B. S. (2015). Sleep and chronic stress. New directions for allostatic load research. Sleep Medicine, 16, 7-8. https://doi.org/10.1016/j.sleep.2014.07.029

Karatsoreos, I. N., \& McEwen, B. S. (2011). Psychobiological allostasis: resistance, resilience and vulnerability. Trends in Cognitive Sciences, 15, 576-584. https://doi.org/10.1016/j.tics. 2011.10.005

Lee, S. W. (2019). A Copernican approach to brain advancement: The paradigm of allostatic orchestration. Frontiers in Human Neuroscience, 13, 129. https://doi.org/10.3389/fnhum.2019.00129

Lunde, C. E., \& Sieberg, C. B. (2020). Walking the tightrope: A proposed model of chronic pain and stress. Frontiers in Neuroscience, 14, 270. https://doi.org/10.3389/fnins.2020.00 270

Mäki-Marttunen, V., Kuusinen, V., Brause, M., Peräkylä, J., Polvivaara, M., dos Santos Ribeiro, R., Öhman, J., \& Hartikainen, K. M. (2015). Enhanced attention capture by emotional stimuli in mild traumatic brain injury. Journal of Neurotrauma, 32, 272-279. https://doi.org/10.1089/neu.2014.3557

McEwen, B. S., \& Gianaros, P. J. (2011). Stress- and allostasisinduced brain plasticity. Annual Review of Medicine, 62, 431445. https://doi.org/10.1146/annurev-med-052209-100430

McEwen, B. S., \& Karatsoreos, I. N. (2015). Sleep deprivation and circadian disruption: Stress, allostasis, and allostatic load. Sleep Medicine Clinics, 10, 1-10. https://doi.org/10.1016/j.jsmc.2014.11.007

McEwen, B. S. (2003). Mood disorders and allostatic load. Biological Psychiatry, 54, 200-207. https://doi.org/10.1016/S00063223(03)00177-X 
McEwen, B. S. (2006). Sleep deprivation as a neurobiologic and physiologic stressor: Allostasis and allostatic load. Metabolism, 55(10 Supp1 2), S20-S23. https://doi.org/10.1016/ j.metabol.2006.07.008

McEwen, B. S. (2012). Brain on stress. How the social environment gets under the skin. Proceedings of the National Academy of Sciences USA, 109(Suppl 2), 17180-17185. https://doi.org/10.1073/pnas.1121254109

McEwen, B. S. (2017). Neurobiological and systemic effects of chronic stress. Chronic Stress, 1, 1-11. https://doi.org/10.1177/ 2470547017692328

McGeary, C., Nabity, P., Reed, D., Cobos, B., Eapen, B., Pugh, M.J., Jaramillo, C., Potter, J., Houle, T., Young-McCaughan, S., Peterson, A., \& McGeary, D. (2020). A test of the fear avoidance model to predict chronic pain outcomes in a polytrauma sample. NeuroRehabilitation, 47, 35-43.

McIntyre, A., Mehta, S., Janzen, S., Rice, D., Harnett, A., MacKenzie, H.M., Vanderlaan, D., \& Teasell, R. (2020a). Coping strategies and personality traits among individuals with brain injury and depressive symptoms. NeuroRehabilitation, 47, 25-34.

McIntyre, A., Rice, D., Janzen, S., Mehta, S., Harnett, A., Caughlin, S., Sequira, K., \& Teasell, R. (2020b). Anxiety, depression, and quality of life among subgroups of individuals with acquired brain injury: The role of anxiety sensitivity and experiential avoidance. NeuroRehabilitation, 47, 45-53.

Mills, S. E. E., Nicholson, K. P., \& Smith, B. H. (2019). Chronic pain. A review of its epidemiology and associated factors in population-based studies. British Journal of Anaesthesiology, 123, e273-e283. https://doi.org/10.1016/j.bja.2019.03.023

Mollayeva, T., Cassidy, J. D., Shapiro, C. M., Mollayeva, S., \& Colantonio, A. (2017). Concussion/mild traumatic brain injury-related chronic pain in males and females: A diagnostic modelling study. Medicine (Baltimore), 96, e5917. https://doi.org/10.1097/MD.0000000000005917

Nampiaparampil, D. E. (2008). Prevalence of chronic pain after traumatic brain injury. A systematic review. Journal of the American Medical Association, 300, 711-719. https://doi.org/10.1001/jama.300.6.711

Peters, A., McEwen, B. S. \& Friston, K. (2017). Uncertainty and stress: Why it causes diseases and how it is mastered by the brain. Progress in Neurobiology, 156, 164-188. https://doi.org/10.1016/j.pneurobio.2017.05.004

Raukola-Lindblom, M., Vuorinen, E., \& Vartiainen, R. (2020). Recovering social participation - experience with a relational group intervention for traumatic brain injury patients. $\mathrm{Neu}$ roRehabilitation, 47, 55-64.

Shaltout, H. A., Lee, S. W., Tegeler, C. L., Hirsch, J. R., Simpson, S. L., Gerdes, L., \& Tegeler, C. H. (2018). Improvements in heart rate variability, baroreflex sensitivity, and sleep after use of closed-loop allostatic neurotechnology by a heterogeneous cohort. Frontiers in Public Health, 6, 116. https://doi.org/0.3389/fpubh.2018.00116

Sterling, P., \& Eyer, J. (1988). Allostasis: a new paradigm to explain arousal pathology. In : Handbook of Life Stress, Cognition and Health, eds S. Fisher and J. Reason. New York, NY: J Wiley \& Sons, pp. 629-649.

Sterling, P. (2012). Allostasis. A model of predictive regulation. Physiology of Behavior, 106, 5-15. https://doi.org/10.1016/ j.physbeh.2011.06.004
Sterling, P. (2014). Homeostasis vs. allostasis: implications for brain function and mental disorders. JAMA Psychiatry, 71, 1192-1193. https://doi.org/10.1001/jamapsychiatry.2014 1043

Strain, J. J. (2018). The psychobiology of stress, depression, adjustment disorders and resilience. World Journal of Biolog ical Psychiatry, 19(supp11), S14-S20. https://doi.org/10.1080/ 15622975.2018.1459049

Tedeschi, R., \& Calhoun, L. (1995). Trauma and Transformation: Growing in the Aftermath of Suffering. Thousand Oaks, CA: Sage.

Tegeler, C. H., Tegeler, C. L., Cook, J. F., Lee, S. W., Gerdes, L., Shaltout, H. A., Miles, C. M., \& Simpson. S. L. (2016). A preliminary study of the effectiveness of an allostatic, closedloop, acoustic stimulation neurotechnology in the treatment of athletes with persisting post-concussion symptoms. Sports Medicine Open, 2, 39. https://doi.org/10.1186/s40798-0160063-y

Vachon-Presseau, E., Roy, M., Martel, M.-O., Caron, E., Marin, M.-F., Chen, J., Albouy, G., Plante, I., Sullivan, M. J., Lupien, S. J., \& Rainville P. (2013). The stress model of chronic pain: evidence from basal cortisol and hippocampal structure and function in humans. Brain, 136(Pt 3), 815-827. https://doi.org/10.1093/brain/aws371

Vachon-Presseau, E., Tétreault, P., Petre, B., Huang, L., Berger, S. E., Torbey, S., Baria, A. T., Mansour, A. R., Hashmi, J. A. Griffith, J. W., Comasco, E., Schnitzer, T. J., Baliki, M. N., \& Apkarian, A. V. (2016). Corticolimbic anatomical characteristics predetermine risk for chronic pain. Brain, 139, 1958-1970. https://doi.org/10.1093/brain/aww100

Vachon-Presseau, E. (2018). Effects of stress on the corticolimbic system: Implications for chronic pain. Progress in Neuropsychopharmacology and Biological Psychiatry, 87(Pt B), 216-223. https://doi.org/10.1016/j.pnpbp.2017.10.014

Walsh, R. S., Crawley, L., Dagnall, N., \& Fortune, D. G. (2020). The man who used to shrug - one man's lived experience of TBI. NeuroRehabilitation, 47, 11-24.

Wickwire, E. M., Schnyer, D. M., Germain, A., Williams, S G., Lettieri, C. J., McKeon, A. B., Scharf, S. M., Stocker, R., Albrecht, J., Badjatia, N., Markowitz, A. J., \& Manley, G. T. (2018). Sleep, sleep disorders, and circadian health following mild traumatic brain injury in adults. Review and research agenda. Journal of Neurotrauma, 35, 2615-2631. https://doi.org/10.1089/neu.2017.5243

Wijenberg, M. L., Stapert, S. Z., Verbunt, J. A., Ponsford, J. L., \& Van Heugten, C. M. (2017). Does the fear avoidance model explain persistent symptoms after traumatic brain injury? Brain Injury, 31, 1597-1604. https://doi.org/10.1080/02699052.2017.1366551

Wolff, M., Evens, R., Mertens, L. J., Koslowski, M., Betzler, F., Grunder, G., \& Jungaberle, H. (2020). Learning to let go: A cognitive-behavioral model of how psychedelic therapy promotes acceptance. Frontiers in Psychiatry, 11, 5. https://doi.org/10.3389/fpsyt.2020.00005

Worley, N. B., Hill, M. N., \& Christianson, J. P. (2018). Prefrontal endocannabinoids, stress controllability and resilience: A hypothesis. Progress in Neuro-psychopharmacology \& Biological Psychiatry, 85, 180-188. https://doi.org/10.1016/ j.pnpbp.2017.04.004 